A N N A L E S Annales de Bretagne et des Pays de l'Ouest

\title{
Les trompettes de la République
}

\section{Loïc Vadelorge}

\section{OpenEdition}

\section{Journals}

Édition électronique

URL : http://journals.openedition.org/abpo/2217

DOI : $10.4000 / a b p o .2217$

ISBN : 978-2-7535-1841-4

ISSN : 2108-6443

\section{Éditeur}

Presses universitaires de Rennes

Édition imprimée

Date de publication : 30 décembre 2011

Pagination : 154-156

ISBN : 978-2-7535-1839-1

ISSN : 0399-0826

\section{Référence électronique}

Loïc Vadelorge, "Les trompettes de la République », Annales de Bretagne et des Pays de l'Ouest [En ligne], 118-4 | 2011, mis en ligne le 30 décembre 2011, consulté le 23 septembre 2020. URL : http:// journals.openedition.org/abpo/2217 ; DOI : https://doi.org/10.4000/abpo.2217

Ce document a été généré automatiquement le 23 septembre 2020.

(c) Presses universitaires de Rennes 


\title{
Les trompettes de la République
}

\author{
Loïc Vadelorge
}

\section{RÉFÉRENCE}

CAMBoN, Jérôme, Les trompettes de la République. Harmonies et fanfares en Anjou sous la Troisième République, Rennes, PUR, 2011, 337 p. (ISBN 978-2-7535-1265-8).

1 Issu d'une thèse de musicologie soutenue en 2009 à l'université François-Rabelais de Tours, Les trompettes de la République constitue un bel exemple de monographie locale, au croisement de l'histoire culturelle et de l'histoire des musiques amateurs. Le propos est précisément centré sur l'histoire des harmonies et fanfares du département du Maine-et-Loire entre 1870 et 1914 , période qui, on le sait, a été très favorable au renouveau du mouvement orphéonique né au cours du premier XIX ${ }^{e}$ siècle.

2 L'ouvrage suit un plan thématique très clair autour de trois axes, social, politique et patrimonial. Dans une première partie, l'auteur revient sur les conditions socioéconomiques qui président au développement des harmonies et fanfares entre le Second Empire et la Belle époque et souligne, après d'autres, le lien intrinsèque existant entre le développement des cultures urbaines et celui de l'orphéon. Dans une seconde partie, il questionne, non sans nuances, l'appropriation du modèle républicain par les sociétés musicales amateurs en comparant les pratiques à l'œuvre dans différentes associations. Dans une troisième et dernière partie enfin, il s'attache aux aspects musicologiques du sujet tant en ce qui concerne l'organisation instrumentale des phalanges qu'en ce qui concerne le répertoire. La lecture est aisée et l'appareil critique précis et précieux.

3 On pourra certes critiquer le plan à tiroir et une tendance trop systématique à compartimenter l'analyse - secteur rural/secteur industriel ou contexte politique/ contexte religieux - mais ce défaut est pour partie compensé par l'incontestable lisibilité de l'ensemble, la table des matières et l'index permettant très rapidement d'accéder au détail de l'information. Celle-ci est d'ailleurs abondante, l'auteur ne négligeant pas de publier de longs extraits d'archives - comptes rendus de la presse 
locale, extraits de délibérations municipales etc. -, des archives photographiques nombreuses et surtout des tableaux récapitulant ses analyses. L'archive est bien mise en avant dans ce travail, ce qui facilitera son utilisation par la communauté des historiens, sociologues et musicologues qui arpentent le terrain de l'histoire des harmonies et des fanfares depuis deux ou trois décennies. La conclusion fait d'ailleurs référence à un certain nombre de travaux récents avec lesquels l'étude est appelée à dialoguer implicitement.

Outre la masse documentaire ici réunie, on soulignera quelques bons passages tant sur la transition entre musique militaire/musique de sapeurs-pompiers et harmoniesfanfares républicaines qu'en ce qui concerne la richesse des fonds musicologiques enfouis dans les archives associatives, municipales et départementales. À cet égard, toute la troisième partie nous semble devoir intéresser les mondes de la conservation bibliothèques, archives - ayant à traiter ce type de fonds, qui posent des problèmes spécifiques. La preuve est en tous cas faite qu'on ne peut plus considérer les partitions des orphéons et, au-delà, les archives des sociétés musicales comme des documents de seconde zone mais qu'elles participent pleinement de l'histoire de la musique occidentale à l'époque contemporaine et doivent être préservées avec soin.

Reste que la publication de cette étude pose au moins deux questions quant à l'évolution même de ce champ de recherche. La première est celle des limites de la pluridisciplinarité sur un tel sujet. Une telle monographie était sans doute nécessaire et acceptable en l'état au début des années 1990, à l'époque où la recherche ne disposait encore que de rares monographies - ainsi celle de Marie-Claire Mussat - et que de quelques études générales - comme celles de Philippe Gumplowicz, de Paul Gerbod et de Danièle Pistone - sur l'histoire de l'orphéon au XIX ${ }^{e}$ siècle. Elle semble aujourd'hui plus délicate à justifier et à mettre en œuvre en embrassant autant d'aspects du sujet, tant l'historiographie a été renouvelée en profondeur par le développement de l'histoire politique et culturelle. La première et la seconde partie de l'ouvrage s'appuient à cet égard sur une historiographie en partie datée. Les travaux de Michèle Alten sur la musique républicaine, de Christine Naslin sur les musiques francmaçonnes, de Ludovic Tournès sur les sociabilités musicales, de Philippe Poirrier sur les politiques culturelles ne sont pas cités, pas plus que les monographies d'histoire culturelle urbaine - sur Bordeaux, Saint-Étienne, Dijon, Rouen etc. - qui ont contribué à restituer l'histoire du mouvement orphéonique dans l'ensemble de l'histoire sociale et culturelle de la Troisième République. De même, les avancées de l'histoire de la mutualité (on pense aux travaux de Bernard Gibaud, d'André Gueslin ou de Michel Dreyfus) ou de l'histoire politique du xix ${ }^{e}$ siècle (on pense à ceux de Pierre Rosanvallon) auraient dû être mobilisées dans les chapitres évoquant les cultures démocratiques et sociales de l'orphéon. Assimiler l'ensemble de cette littérature n'était sans doute pas possible dans le temps nécessairement limité consacré à la monographie, qui a sciemment privilégié - et à juste titre - l'analyse du matériel archivistique. Reste que l'ouvrage pèche par endroits - dans les première et deuxième parties notamment - en proposant des conclusions sur la sociologie ou la culture politique des orphéons qui mériteraient davantage de nuances et de contextualisation.

6 La seconde question que nous semble poser cet ouvrage est celle de la pertinence de l'approche départementale. On se souvient de l'appel lancé jadis par Jacques Rougerie dans les Annales: «Faut-il départementaliser l'histoire de France?» La lecture des Trompettes de la République conduit à se poser la même question. L'échelle 
départementale est-elle la bonne échelle d'analyse des pratiques musicales amateurs sous la Troisième république? Jérôme Cambon a eu nettement conscience de cette difficulté qu'il résout en partie de deux manières complémentaires. Il démontre d'une part (voir les très bonnes cartes de la page 310) que le Maine-et-Loire a une particularité géographique puisqu'il est composé d'un réseau de villes moyennes Angers, Saumur, Cholet, Segré, Baugé - qui favorisent la diffusion de l'orphéon. Il démontre d'autre part que l'histoire musicale de ce département a été longtemps sousestimée par la recherche et qu'une réhabilitation était nécessaire tant sous l'angle quantitatif que qualitatif. Dans les deux cas, la démonstration justifie le recours à la monographie. Reste que l'échelle départementale fonctionne moins bien pour certains sujets abordés - les liens entre orphéon et mutualité, le répertoire, la facture instrumentale - qui relèvent d'une échelle régionale ou nationale. Reste aussi et surtout que le contexte départemental n'est paradoxalement pas assez fouillé. Quid de l'évolution politique du conseil général, de la prosopographie des préfets, dont l'auteur démontre le rôle essentiel aux côtés des maires dans le développement de la musique amateur? Quid des rapports avec l'Église - les maîtrises - et les milieux conservateurs au sein de ce département situé aux portes de la Bretagne? Quid des rapports avec les sociétés savantes et les notables? Ces points sont certes évoqués mais trop rapidement, au détour d'un paragraphe ou d'une note de bas de page, lors même qu'ils semblaient essentiels pour creuser la question de la spécificité des pratiques musicales dans ce département de l'Ouest.

7 Au total, cet ouvrage, publié avec beaucoup de rigueur, nous apparaît comme une contribution utile pour une histoire musicale de la France contemporaine soucieuse de s'ouvrir à toutes les pratiques et écritures de la musique. Parce qu'il aborde les questions générales de l'histoire sociale et musicale de l'orphéon, il dépasse l'intérêt strictement local et mérite en ce sens une large diffusion. Mais sans doute est-il à présent nécessaire de dépasser ce type d'approche monographique. Les travaux à venir devront davantage s'employer à réduire la focale, à élargir l'échelle de référence et à mieux répartir ce qui relève de la responsabilité des historiens et de celle des musicologues.

LOḮ VADELORGE 